\title{
FULL-WAVEFORM INVERSION OF SH-WAVE INPUT MOTIONS IN A NEAR-SURFACE 2D DOMAIN
}

\author{
BRUNO P. GUIDIO ${ }^{1}$ AND CHANSEOK JEONG ${ }^{1}$ \\ ${ }^{1}$ School of Engineering and Technology, Central Michigan University \\ Mount Pleasant, MI 48859, USA \\ peruq1b@cmich.edu (the presenting author) \& jeong1c@cmich.edu (the corresponding author)
}

Key words: PDE-constrained Optimization, Adjoint Method, Incoherent Dynamic Traction Inversion, Absorbing Boundary Condition

\begin{abstract}
There is a need to estimate complex seismic input motions in a near-surface domain, without resorting to the hypocenter, from restricted seismic measurement data. Thus, engineers can replicate responses within structures and soils after an earthquake occurrence by using the estimated seismic inputs and evaluate the impact of an earthquake on the built environment. To date, there has been no robust numerical method that can identify complex seismic input motions in a solid, truncated by a wave-absorbing boundary condition. Existing methods are limited to large-scale seismic-source inversion approaches and deconvolution. To fill this gap, a new inversion modeling method is presented for reconstructing complex, incoherent SH wave input motions in a two-dimensional (2D) domain that is truncated by a wave-absorbing boundary condition (WABC), using a partial differential equation (PDE)constrained optimization method. In a set of numerical examples, targeted, dynamic traction at the WABC mimics seismic incident wavefield. The discretize-then-optimize (DTO) approach is used in the mathematical modeling and numerical implementation, and the finite element method (FEM) is applied to solve state and adjoint problems. The numerical results indicate that the presented inversion algorithm can reconstruct incident, inclined plane waves. Second, the accuracy of our inversion solver is not compromised by the material complexity of a background domain. Lastly, the minimizer suffers less from solution multiplicity when it identifies lower frequency traction (e.g., a realistic seismic signal).
\end{abstract}

\section{INTRODUCTION}

To date, there has been no robust numerical method that can identify complex seismic input motions in a solid, truncated by a wave-absorbing boundary condition. Existing methods are limited to largescale seismic-source inversion approaches [1] and deconvolution [14, 29-31]. As an alternative to such conventional methods, this paper presents a new numerical method to identify arbitrary, incoherent incoming seismic waves in a multi-dimensional truncated domain using sparse seismic measurement. To this end, we discretize the spatial and temporal distribution of an estimated incident wave and calculate the discretized parameters of the estimated wave that can minimize the discrepancy between the measured data at sensor locations and their computed counterparts induced by the estimated wave. The PDE-constrained optimization framework has advanced the inverse problems associated with elastodynamic wave motions, and the associated applications span from material and scatterer characterization to dynamic-input identification. For example, the geotechnical site characterization methodologies have 


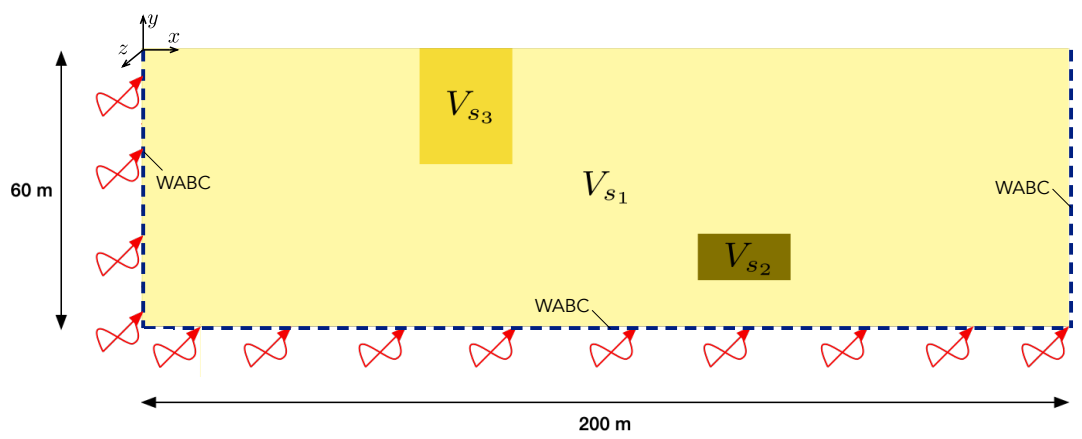

Figure 1: The problem configuration, showing an undamped homogeneous domain with its wave speed $V_{s_{1}}$ and inclusions with their wave speeds $V_{s_{2}}$ and $V_{s_{3}}$.

been investigated in a truncated soil domain $[2-7,12,15-19,23-26,28]$. The inversion approach had also been used for estimating the most desirable, non-moving wave source profiles that can maximize wave energy in specific areas in solids [9-11, 20-22, 32]. To investigate the feasibility to identify arbitrary, incoherent incoming seismic waves in a truncated domain by using sparse seismic measurement by using the PDE-constrained optimization framework, $[8,13]$ studied a full-waveform source-inversion method to identify an incoming seismic wave in a 1D semi-infinite solid and a 2D bounded domain. Continuing the aforementioned papers $[8,13]$, the presented paper discusses a numerical method that can fully reconstruct the comprehensive profiles of complex, incoherent seismic incident wavefields propagating into a 2D truncated domain of anti-plane shear wave motion

\section{GOVIENING WAVE PHYSICS}

Fig. 1 displays the problem configuration where

absorbing boundary condition (WABC), and the

impinges the domain with an incident angle of 45
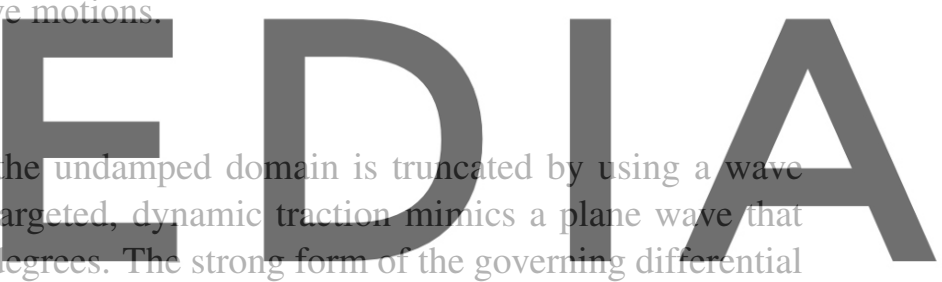

equation for the shear wave propagation in the domain $\Omega$, for time $t \in J=(0, T]$, is:

Register for free at https//www.scipedia.comzto download the version without the watermark

$$
\nabla \cdot(G \nabla u)-\rho \frac{\partial^{2} u}{\partial t^{2}}=0, \quad \text { on } \Omega \times J,
$$

where $u=u(x, y, t)$ denotes the displacement field in the anti-plane $(z)$ direction of the wave motion of a solid particle; $x$ and $y$ denote the horizontal and vertical coordinates; $G(x, y)$ and $\rho(x, y)$ denote the shear modulus and the mass density of the solid.

The traction-free condition is presented on the top surface $\left(\Gamma_{\mathrm{t}}\right)$, while the absorbing boundary conditions are presented on the bottom $\left(\Gamma_{\mathrm{b}}\right)$, left $\left(\Gamma_{1}\right)$ and right $\left(\Gamma_{\mathrm{r}}\right)$ boundaries:

$$
\begin{aligned}
\frac{\partial u}{\partial y}(x, 0, t)=0, & 0 \leq x \leq L, \\
\frac{\partial u}{\partial y}(x, D, t)=\frac{f(x, D, t)}{G}-\frac{1}{V_{\mathrm{s}}} \frac{\partial u}{\partial t}, & 0 \leq x \leq L, \\
\frac{\partial u}{\partial x}(0, y, t)=\frac{f(0, y, t)}{G}-\frac{1}{V_{\mathrm{s}}} \frac{\partial u}{\partial t}, & D \leq y \leq 0, \\
\frac{\partial u}{\partial x}(L, y, t)=-\frac{1}{V_{\mathrm{s}}} \frac{\partial u}{\partial t}, & D \leq y \leq 0,
\end{aligned}
$$


where $D$ is the $y$-coordinate of $\Gamma_{\mathrm{b}} ; L$ is the $x$-coordinate of $\Gamma_{\mathrm{r}}$; and $V_{\mathrm{s}}(x, y)$ is the shear wave speed of the medium. We note that $f(x, y, t)$ denotes the dynamic traction of shear stress applied in the out-of-plane direction on the boundaries. Finally, the initial conditions are:

$$
u(x, y, 0)=0, \quad \frac{\partial u}{\partial t}(x, y, 0)=0
$$

\section{NUMERICAL MODELING}

\subsection{Finite element}

The weak form of the forward problem is cast by multiplying the governing equation (1) by the test function $v$ :

$$
\begin{array}{r}
\int_{\Omega} \nabla v \cdot(G \nabla u) \mathrm{d} \Omega+\int_{\Gamma_{\mathrm{r}}} v \frac{G}{V_{\mathrm{S}}} \frac{\partial u}{\partial t} \mathrm{~d} \Gamma+\int_{\Gamma_{1}} v \frac{G}{V_{\mathrm{S}}} \frac{\partial u}{\partial t} \mathrm{~d} \Gamma+\int_{\Gamma_{\mathrm{b}}} v \frac{G}{V_{\mathrm{S}}} \frac{\partial u}{\partial t} \mathrm{~d} \Gamma \\
+\int_{\Omega} v \rho \frac{\partial^{2} u}{\partial t^{2}} \mathrm{~d} \Omega=\int_{\Gamma_{\mathrm{b}}} v f(x, y, t) \mathrm{d} \Gamma+\int_{\Gamma_{1}} v f(x, y, t) \mathrm{d} \Gamma,
\end{array}
$$

Then, by introducing the finite element approximations of the trial and test functions, the governing wave physics yields to the following discrete system:
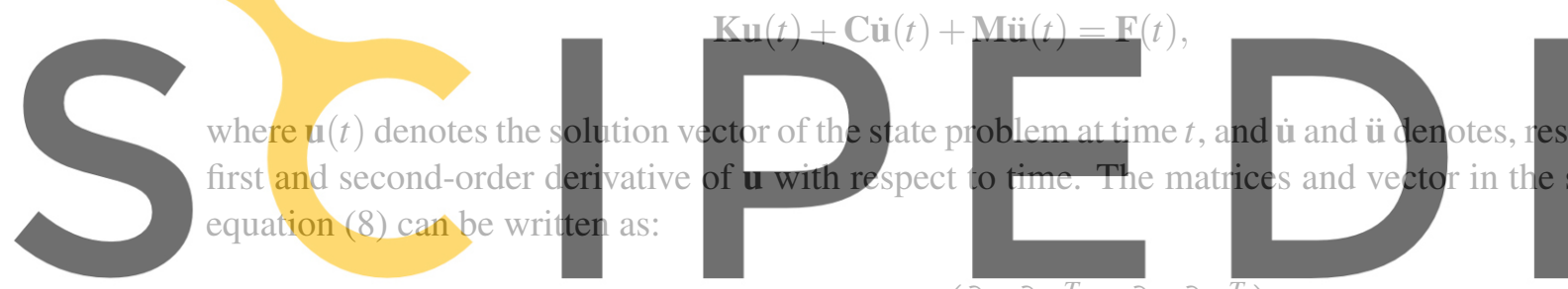

$$
\mathbb{K}=\int G\left(\frac{\partial \psi}{\partial y} \frac{\partial \psi^{T}}{\partial x}+\frac{\partial \psi}{\partial \psi} \frac{\partial \psi^{T}}{\partial r^{T}}\right) \mathrm{d} \Omega
$$

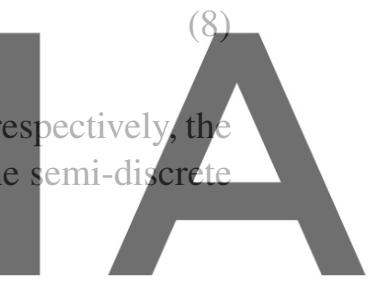

Register for free at https//www.scipedia.com to dyownloald the version without the watermark

$$
\begin{array}{r}
\mathrm{C}=\int_{\Gamma_{\mathrm{r}}} \frac{G}{V_{\mathrm{S}}} \psi \psi^{T} \mathrm{~d} \Gamma+\int_{\Gamma_{1}} \frac{G}{V_{\mathrm{S}}} \psi \psi^{T} \mathrm{~d} \Gamma+\int_{\Gamma_{\mathrm{b}}} \frac{G}{V_{\mathrm{S}}} \psi \psi^{T} \mathrm{~d} \Gamma, \\
\mathbf{M}=\int_{\Omega} \rho \psi \psi^{T} \mathrm{~d} \Omega, \\
\mathbf{F}=\int_{\Gamma_{\mathrm{b}}} \psi f \mathrm{~d} \Gamma+\int_{\Gamma_{1}} \psi f \mathrm{~d} \Gamma .
\end{array}
$$

\subsection{Compact form}

We solve (8) by using the implicit Newmark time integration. Although the time-integration steps are omitted, the procedure can be summarized in the following equation:

$$
\mathbf{Q} \hat{\mathbf{u}}=\hat{\mathbf{F}},
$$

where the vector $\hat{\mathbf{u}}$ corresponds to the solution vector to all the time steps (i.e., the space-time discretization of $u(x, y, t)$ ); and $\hat{\mathbf{F}}$ is the global force vector. These vectors are built as: 


$$
\hat{\mathbf{u}}=\left[\begin{array}{c}
\mathbf{u}_{0} \\
\dot{\mathbf{u}}_{0} \\
\ddot{\mathbf{u}}_{0} \\
\vdots \\
\mathbf{u}_{\mathrm{N}} \\
\dot{\mathbf{u}}_{\mathrm{N}} \\
\ddot{\mathbf{u}}_{\mathrm{N}}
\end{array}\right], \quad \hat{\mathbf{F}}=\left[\begin{array}{c}
0 \\
0 \\
\mathbf{F}_{0} \\
\vdots \\
\mathbf{F}_{\mathrm{N}} \\
0 \\
0
\end{array}\right],
$$

where $\mathrm{N}$ is the number of time steps, and $\mathbf{u}_{j}$ are the spatial degrees of freedom at the $j$-th time step. Finally, the matrix $\mathbf{Q}$ is defined as:

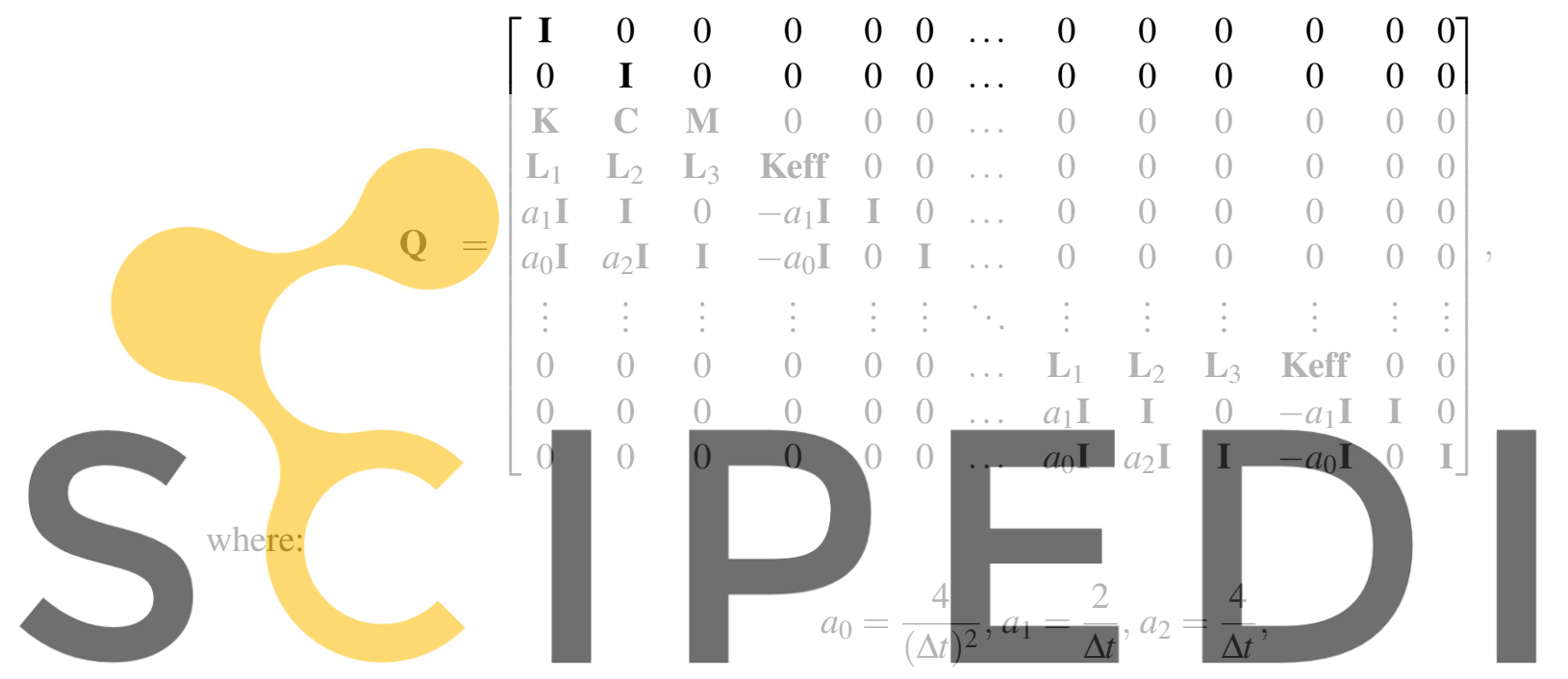

\section{$4 \quad$ INVERSE MODELING}

The presented work studies the discretize-then-optimize (DTO) approach. Under this inversion method, we identify $P_{k j}$-a component of $\hat{\mathbf{F}}$ corresponding to $\gamma_{k}$ and $t_{j}$ - as control parameters, where $\gamma_{k}$ is the $k$-th discrete node on the boundaries $\Gamma_{1}$ and $\Gamma_{\mathrm{b}}$. The discrete numbering of $\gamma_{k}$ increases counter-clockwise from the top-left corner of the domain, and $t_{j}$ is the $j$-th time step.

\subsection{Discrete objective and Lagrangian functional}

As a minimization problem, we attempt to determine the values of $P_{k j}$ that minimize the discrete objective functional, which is defined as:

$$
\hat{\mathcal{L}}=\frac{1}{2}\left(\hat{\mathbf{u}}-\hat{\mathbf{u}}_{\mathrm{m}}\right)^{T} \overline{\mathbf{B}}\left(\hat{\mathbf{u}}-\hat{\mathbf{u}}_{\mathrm{m}}\right)
$$

where $\hat{\mathbf{u}}$ is obtained by a set of estimated $P_{k j} ; \hat{\mathbf{u}}_{m}$ (i.e., the space-time discretization of $u_{\mathrm{m}}(x, y, t)$ induced by a set of $P_{k j}$ corresponding to targeted force vector) is artificially generated by using a pseudo target of 
traction. In (14), $\overline{\mathbf{B}}$ is defined as $\Delta t \mathbf{B}$, where $\mathbf{B}$ is a square matrix, of which components are all 0 except for those of the diagonal, having values of all 1 , if they correspond to the degrees of freedom of sensor locations. In this work, the measured data is obtained from sparsely-distributed sensors.

To tackle the minimization problem, we use the Lagrange multiplier vector $\hat{\lambda}$ to impose (10) onto (14), casting the following Lagrangian functional:

$$
\hat{\mathcal{A}}=\frac{1}{2}\left(\hat{\mathbf{u}}-\hat{\mathbf{u}}_{\mathrm{m}}\right)^{T} \overline{\mathbf{B}}\left(\hat{\mathbf{u}}-\hat{\mathbf{u}}_{\mathrm{m}}\right)-\hat{\lambda}^{T}(\mathbf{Q} \hat{\mathbf{u}}-\hat{\mathbf{F}})
$$

where $\hat{\lambda}$ is built as:

$$
\hat{\lambda}=\left[\lambda_{0}^{T}, \dot{\lambda}_{0}^{T}, \ddot{\lambda}_{0}^{T}, \ldots, \lambda_{N}^{T}, \dot{\lambda}_{N}^{T}, \ddot{\lambda}_{N}^{T},\right]^{T}
$$

\subsection{Optimality conditions}

To identify unknown target control parameters, the first-order optimality conditions should be fulfilled. The first condition, $(\partial \hat{\mathcal{A}} / \partial \hat{\lambda})=0$, will be automatically satisfied when we solve the discrete forward problem (10), which is also referred to as the discrete state equation:

$$
\frac{\partial \hat{\mathcal{A}}}{\partial \hat{\lambda}}=-\mathbf{Q} \hat{\mathbf{u}}+\hat{\mathbb{F}}=0 .
$$
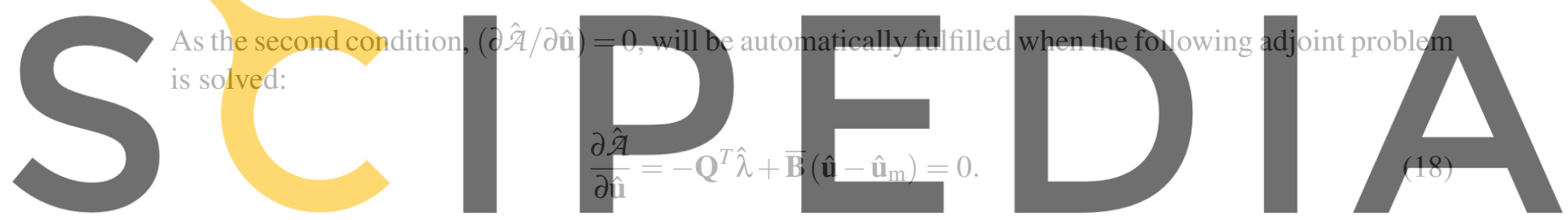

We note that in order to solve the adjoint equation (18), we have to march backward in time because it Register forlffree at. https//www.scipedia.com to download the version without the watermark

As the third condition, $(\partial \hat{\mathfrak{A}} / \partial \hat{\mathbb{F}})=0$, will be satisfied when we solve the resulting control problem:

$$
\frac{\partial \hat{\mathcal{A}}}{\partial \hat{\mathbf{F}}}=\hat{\lambda}=0
$$

which implies that the inversion solution of $\hat{\mathbf{F}}$ should lead to the vanishing $\hat{\lambda}$. (19) also implies that $\frac{\partial \hat{\mathcal{A}}}{\partial \xi}=\frac{\partial \hat{\mathcal{L}}}{\partial \xi}$ is the component of the vector $\hat{\lambda}$ corresponding to the global node numbering and the time step of $\xi$. In other words, if $\xi$ is $P_{k j}$, (19) becomes:

$$
\nabla_{\left(\xi=P_{k j}\right)} \hat{\mathcal{A}}=\nabla_{\left(\xi=P_{k j}\right)} \hat{\mathcal{L}}= \begin{cases}\ddot{\lambda}\left(\gamma_{k}, t_{j}\right) & \text { when } j=1, \\ \lambda\left(\gamma_{k}, t_{j}\right) & \text { when } j \geq 2,\end{cases}
$$

where $\ddot{\lambda}\left(\gamma_{k}, t_{j}\right)$ or $\lambda\left(\gamma_{k}, t_{j}\right)$ denotes the component of $\hat{\lambda}$ corresponding to the degree of freedom and the timestep of $P_{k j}$. 


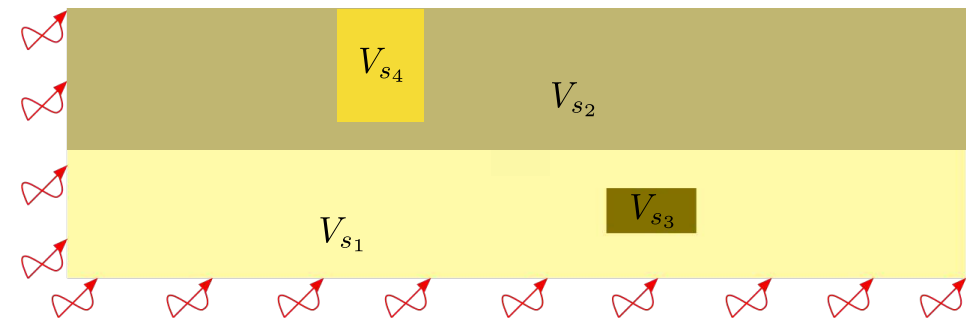

Figure 2: Material profile 2: a 2-layered solid with two inclusions.

\subsection{Control parameters updates}

In a gradient-based scheme, similar to [8], the control parameter vector $\xi$ consisting of $P_{k j}$ is updated iteratively as follows:

$$
\xi_{(s+1)}=\xi_{(s)}+h_{(s)} \mathbf{d}_{(s)},
$$

where $h_{(s)}$ is a step length and $\mathbf{d}_{(s)}$ is a search-direction vector for $\xi_{(s)}$, and $s$ is the inversion iteration index. Moreover, in every inversion iteration, Newton's method $[8,27]$ is utilized to establish the optimal $h_{(s)}$

\section{NUMERICAL EXPERIMENTS}
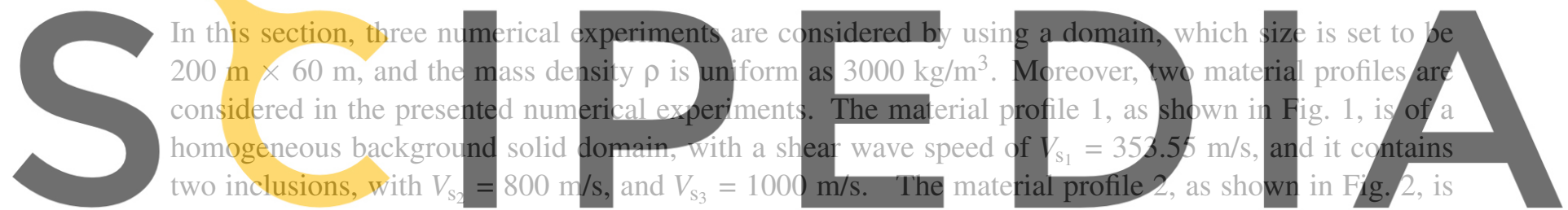
two inclusions, with $V_{s_{2}}=800 \mathrm{~m} / \mathrm{s}$, and $V_{s_{3}}=100$ e

a 2-layered background solid with the same two inclusions. The shear wave speeds of material profile 2

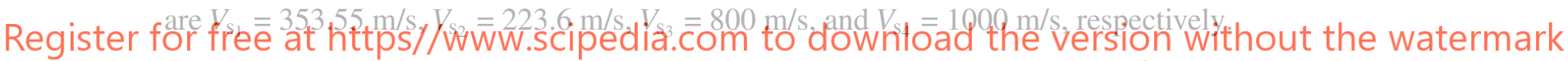

The element size is set to be $0.5 \mathrm{~m}$ for computing the synthetic measured data $\hat{u}_{\mathrm{m}}$, while an element size of $1 \mathrm{~m}$ is used for evaluating state solution $\hat{\mathbf{u}}$ and the adjoint solution $\hat{\lambda}$ for an estimated force vector.

However, the same $\Delta \mathrm{t}$ of $0.001 \mathrm{~s}$ is used for computing all $\hat{\mathbf{u}}_{\mathrm{m}}, \hat{\mathbf{u}}$, and $\hat{\lambda}$.

For the material profile 1, two targeted force vectors $\hat{\mathbf{F}}^{1,3}$ (corresponding to two targeted, dynamic tractions applied on the left and bottom boundaries of the domain) are considered in this section. The time-dependent values of targeted $\hat{\mathbf{F}}^{1}$ corresponding to a node at a specific location $x=100 \mathrm{~m}$ and $y=$ $-60 \mathrm{~m}$ and its frequency content $-\mathrm{a}$ Ricker wavelet with with a central frequency of $5 \mathrm{~Hz}-$ are shown in Fig. 3(a-b). The time-dependent signal corresponding to $\hat{\mathbf{F}}^{3}$ at the same location, and its frequency content is shown in Fig. 3(c-d). This six-second length signal was extracted from the ground motion database of the Pacific Earthquake Engineering Research Center (PEER), with this portion being recorded during the 1994 Northridge earthquake.

On the other hand, $\hat{\mathbf{F}}^{2}$ is a force vector due to dynamic traction on the left and bottom boundaries of a two-layered background domain (i.e., the material profile 2), and this signal mimics incident plane wavefield of an incident angle $45^{\circ}$ in the bottom layer. The signal corresponding to $\hat{\mathbf{F}}^{2}$ at $x=100 \mathrm{~m}$ and $y=-60 \mathrm{~m}$ is a Ricker wavelet with a central frequency of $5 \mathrm{~Hz}$. 

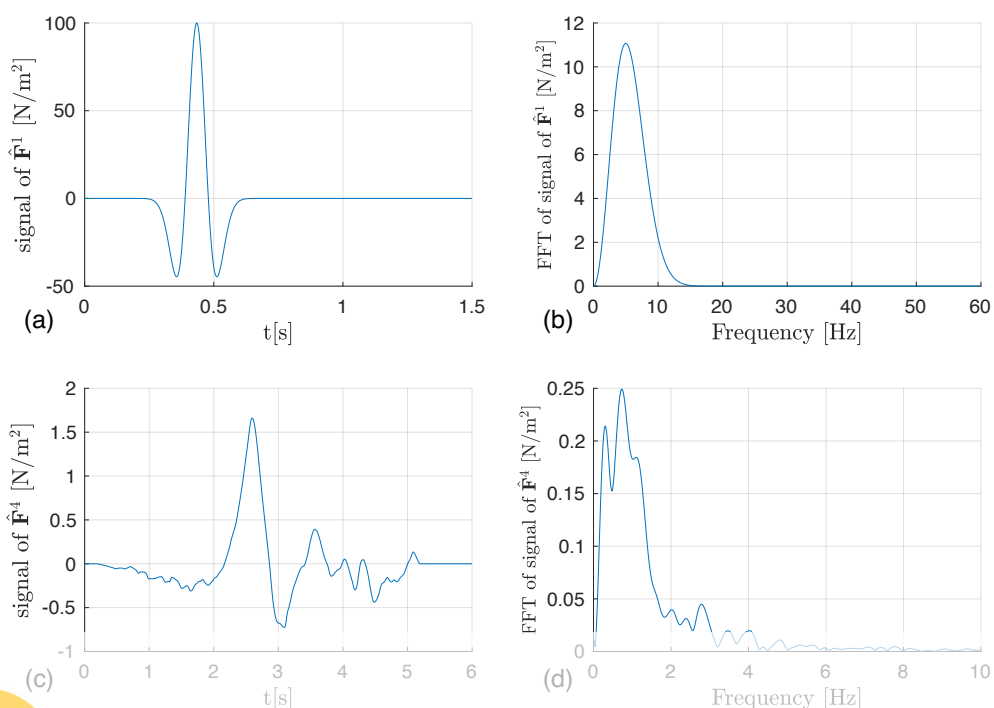

Figure 3: (a,c) The time signals of $\hat{\mathbf{F}}^{1,3}$ corresponding to a node at $x=100 \mathrm{~m}$ and $y=-60 \mathrm{~m}$, respectively; (b,d) the amplitude of Fourier Transform of each signal.

Cases 1 and 2, which consider $\hat{\mathbf{F}}^{1,2}$, use 391,791 control parameters, while Case 3, which consider $\hat{\mathbf{F}}^{3}$, uses $1,566,261$ control parameters. Prior to the first inversion iteration, the control parameters are set as
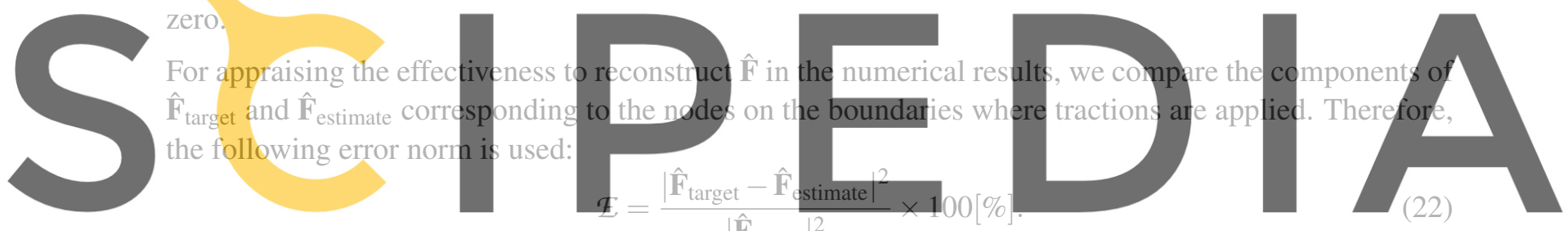

Register for free at https/fwww. scipedia, com to download the version without the watermark

In this example, the performance of inverting a targeted set of $P_{k j}$ of $\hat{\mathbb{F}}^{1}$ by considering a single-layered background domain with inclusions (i.e., Case 1) is compared with the performance of inverting a targeted set of $P_{k j}$ of $\hat{\mathbf{F}}^{2}$ using the two-layered, heterogeneous background domain counterpart (i.e., Case 2). To this end, the traction signals of both $\hat{\mathbf{F}}^{1}$ and $\hat{\mathbf{F}}^{2}$ have the same central frequency of $5 \mathrm{~Hz}$, and the same sensor configuration is used in both Cases- 50 sensors on $\Gamma_{t}$ and a vertical array of 15 sensors on $\Gamma_{r}$. The great agreement between the targeted $P_{k j}$ and its estimated equivalent is indicated in Fig. 4 for both Cases. Fig. 5 shows the value of $\mathcal{E}$ over iterations in Case 1 and 2 for material profiles 1 and 2, respectively. Despite using a more complex background domain in Case 2, the difference between the terminal values of $\mathcal{E}$ in Cases 1 and 2 is only $0.11 \%$.

Thus, we suggest that the accuracy of our presented inversion algorithm is not compromised by the material complexity of a background domain.

\subsection{Example 2: Inversion performance with respect to the signal complexity.}

This example investigates the performance of identifying a targeted set of $P_{k j}$ of $\hat{\mathbf{F}}^{3}$, which is created 

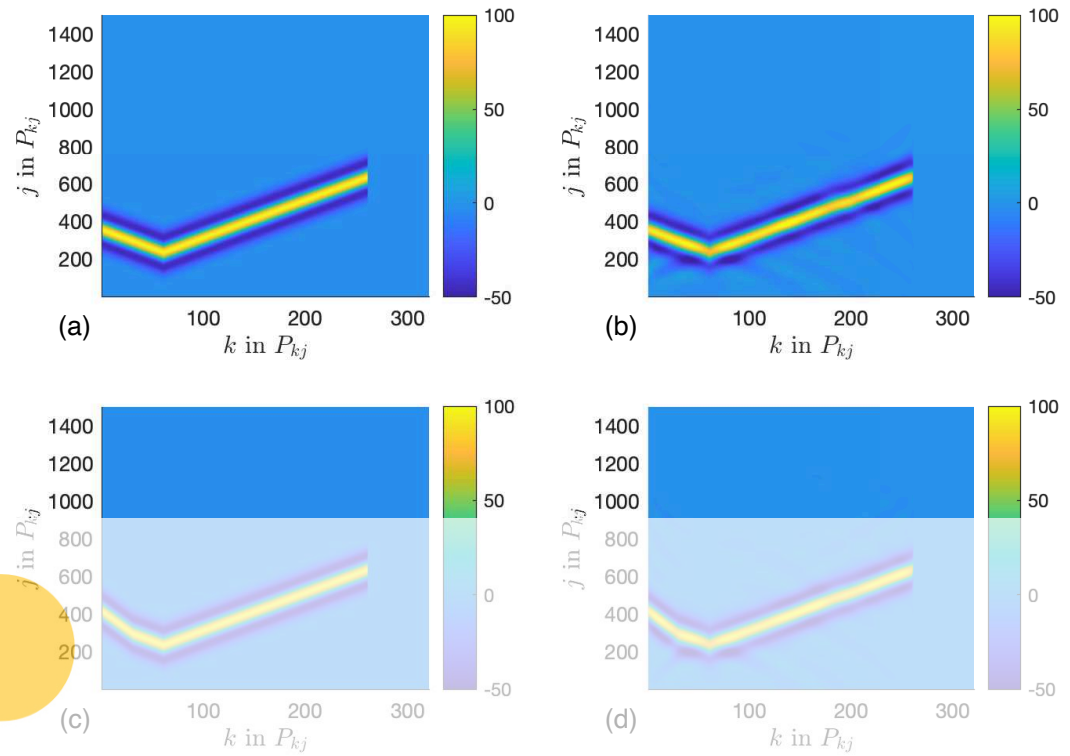

Figure 4: Example 1: (a) Target and (b) Reconstructed $P_{k j}$ for Case 1; and (c) Target and (d) Reconstructed $P_{k j}$ for Case 2 at the 1000th iteration.
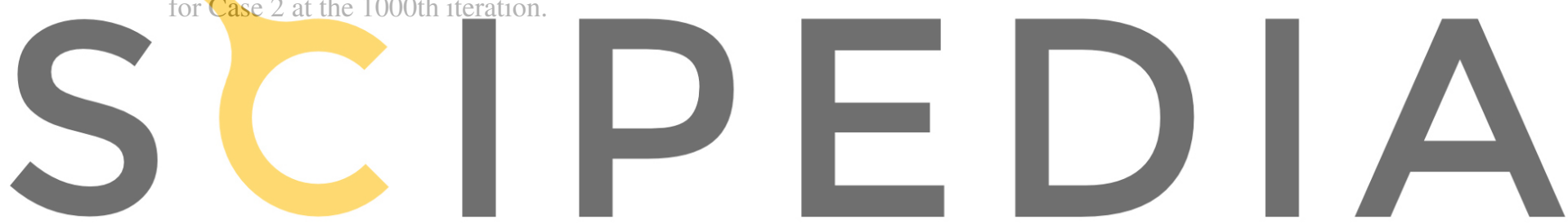

Register for free at https//www.scipedia.com to download thetversion without the watermark

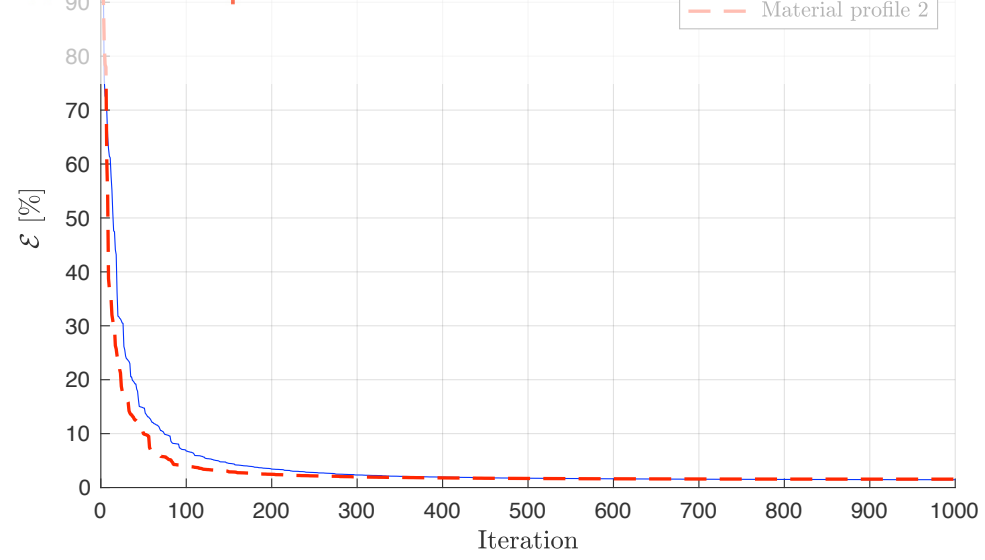

Figure 5: Example 1 - The final value of $\mathcal{E}$, in Case 1, is 1.45\%, and that in Case 2 is $1.56 \%$. 

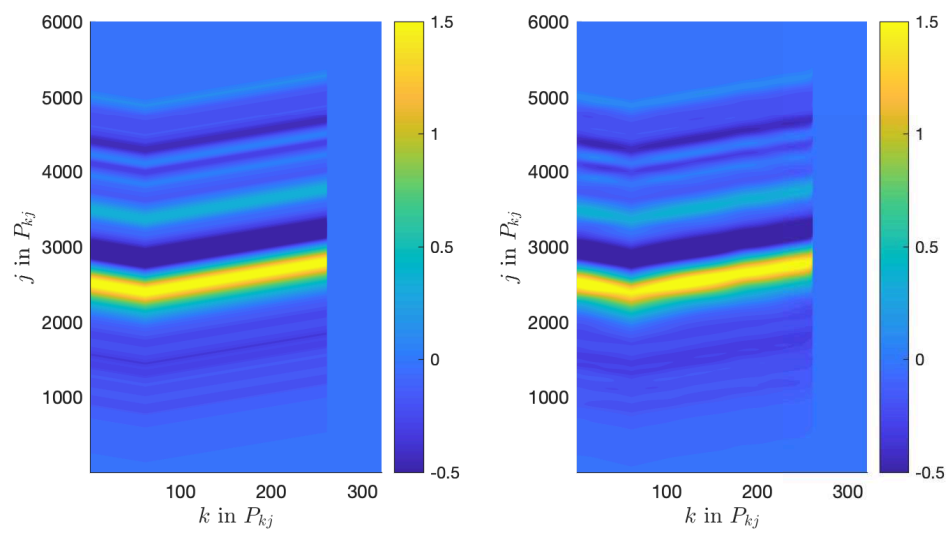

Figure 6: Example 2: (a) A target set of $P_{k j}$ and (b) its reconstructed counterpart corresponding to $\hat{\mathbf{F}}^{2}$ for Case 3 at the 1000 th iteration.
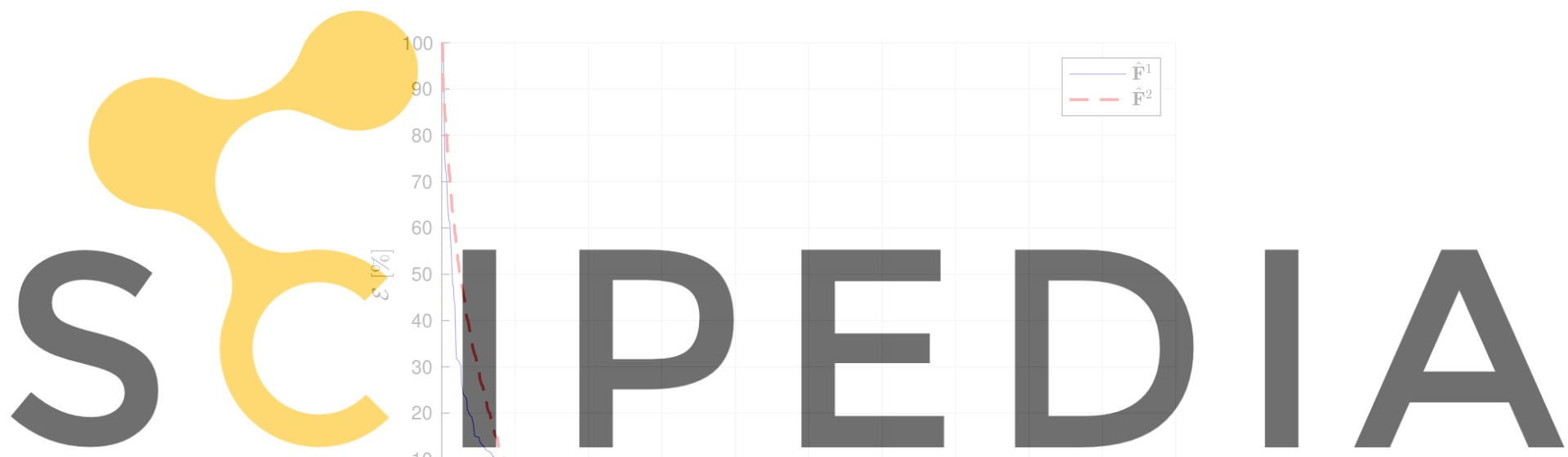

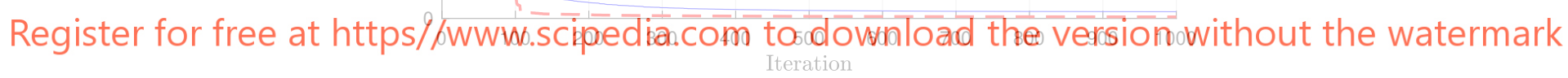

Figure 7: Example 2 - E over iterations in Case 1 and Case 2.

by a realistic seismic signal (Case 3). In Case 3, we use the material profile 1 with 50 sensors on $\Gamma_{t}$ and 15 sensors on $\Gamma_{r}$. Fig. 6 compares the targeted and reconstructed $P_{k j}$ in Case 3, and it shows the excellent concordance between them. The inversion performance in Case 3 is also compared with that in Case 1. Cases 1 and 3 use the same material and sensor configurations except for different force vectors $\hat{\mathbf{F}}^{1}$ and $\hat{\mathbf{F}}^{3}$, respectively. Fig. 7 shows that the final value of $\mathcal{E}, 0.33 \%$, in Case 3 is smaller than that in Case $1(1.45 \%)$. We note that, even though the signal to generate $\hat{\mathbf{F}}^{3}$ is more complex than the other corresponding to $\hat{\mathbf{F}}^{1}$, our inversion solver identifies $\hat{\mathbf{F}}^{3}$ more accurately than $\hat{\mathbf{F}}^{1}$ because the signal in $\hat{\mathbf{F}}^{3}$ has a lower dominant frequency than $\hat{\mathbf{F}}^{1}$. Thus, we suggest that the presented inverse modeling experiences less solution multiplicity when it reconstructs lower frequency traction (e.g., a realistic seismic signal), as discussed in [8]. 


\section{CONCLUSIONS}

In this study, we used a PDE-constrained optimization method to reconstruct incoherent dynamic traction in a 2D domain truncated by wave-absorbing boundary conditions (WABC). Specifically, we used the dynamic traction at the WABC to mimic seismic incident wavefield, the optimization method is utilized to tackle the inverse problem, and the DTO approach is employed to solve the adjoint problem. It was shown that the target traction can be reconstructed by using the presented inverse method. We also showed that the traction signals are well reconstructed despite the complexity of the background domain. Additionally, we showed that the presented minimizer can reconstruct a realistic seismic signal, and it suffers less when identifying signals with a lower frequency content.

\section{References}

[1] Akcelik, V., Biros, G., And Ghattas, O. Parallel multiscale Gauss-Newton-Krylov methods for inverse wave propagation. In Supercomputing, ACM/IEEE 2002 Conference (2002), IEEE, pp. 41-41.

[2] Askan, A., Akcelik, V., Bielak, J., And Ghattas, O. Full Waveform Inversion for Seismic Velocity and Anelastic Losses in Heterogeneous Structures. Bulletin of the Seismological Society of America 97, 6 (12 2007), 1990-2008.

[3] Askan, A., Akcelik, V., Bielak, J., And Ghattas, O. Parameter sensitivity analysis of a nonlinear least-squares optimization-based anelastic full waveform inversion method. Comptes

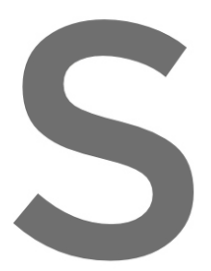
Rendus Mécaniques

[4] Askan, A., AND BIELAK, J. F I Bulletin of the Seism

5] FAthi, A., Kallivokas dimensional PML-truncated elastic media.
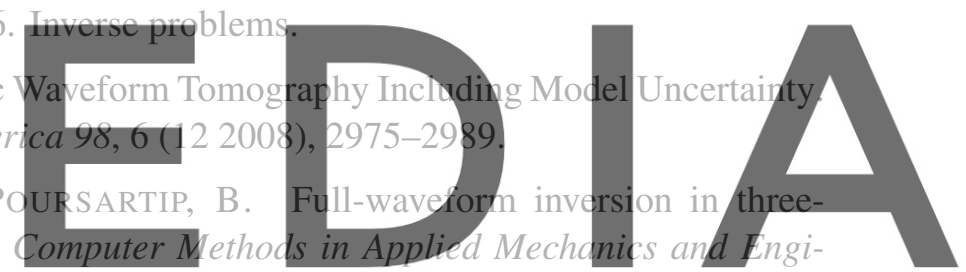
neering 296 (2015), 39-72.

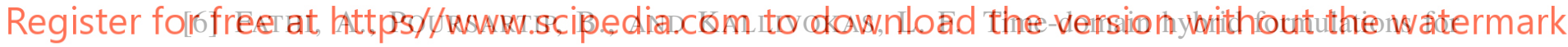
wave simulations in three-dimensional PML-truncated heterogeneous media. International Journal for Numerical Methods in Engineering 101, 3 (2015), 165-198.

[7] Fathi, A., Poursartip, B., Stokoe II, K. H., And Kallivokas, L. F. Three-dimensional P-and S-wave velocity profiling of geotechnical sites using full-waveform inversion driven by field data. Soil Dynamics and Earthquake Engineering 87 (2016), 63-81.

[8] Guidio, B. P., And Jeong, C. Full-waveform inversion of incoherent dynamic traction in a bounded 2d domain of scalar wave motions. Journal of Engineering Mechanics 147, 4 (2021), 04021010 .

[9] Jeong, C., Kallivokas, L., Kucukcoban, S., Deng, W., and Fathi, A. Maximization of wave motion within a hydrocarbon reservoir for wave-based enhanced oil recovery. Journal of Petroleum Science and Engineering 129 (2015), 205-220.

[10] Jeong, C., AND Kallivokas, L. F. An inverse-source problem for maximization of pore-fluid oscillation within poroelastic formations. Inverse Problems in Science and Engineering (2016). 
[11] Jeong, C., Kallivokas, L. F., Huh, C., and Lake, L. W. Optimization of sources for focusing wave energy in targeted formations. Journal of Geophysics and Engineering 7 (2010), 242-256.

[12] JeOng, C., NA, S.-W., AND Kallivokas, L. F. Near-surface localization and shape identification of a scatterer embedded in a halfplane using scalar waves. Journal of Computational Acoustics 17, 03 (2009), 277-308.

[13] JEONG, C., AND SEYlabi, E. E. Seismic input motion identification in a heterogeneous halfspace. Journal of Engineering Mechanics 144, 8 (2018), 04018070.

[14] JU, S. H. A Deconvolution scheme for determination of seismic loads in finite element analyses. Bulletin of the Seismological Society of America 103, 1 (Feb. 2013), 258-267.

[15] Jung, J., JEONG, C., AND TACIROGLU, E. Identification of a scatterer embedded in elastic heterogeneous media using dynamic XFEM. Computer Methods in Applied Mechanics and Engineering 259 (2013), 50-63.

[16] Kallivokas, L. F., Fathi, A., Kucukcoban, S., Stokoe, K. H., Bielak, J., and GhatTAS, O. Site characterization using full waveform inversion. Soil Dynamics and Earthquake Engineering 47 (2013), 62-82.

[17] Kang, J. W., And Kallivokas, L. F. The inverse medium problem in 1D pml-truncated heterogeneous semi-infinite domains. Inverse Problems in Science and Engineering 18, 6 (2010), $759-786$.

[18] Kang, J. W., AND KallivoKas, L. F. Mixed unsplit-field perfectly matched layers for transient simulations of scalar waves in heterogeneous domains. Computational Geosciences 14, 4 (2010), 623-648.

[19] Kang, J. W., And Kallivokas, L. F. The inverse medium problem in heterogeneous PMLtruncated domains using scalar probing waves. Computer Methods in Applied Mechanics and Engineering 200, 1 (2011), 265 - 283.

[20] Karve, P. M., AND KAllivokAs, L. F. Wave energy focusing to subsurface poroelastic formations to promote oil mobilization. Geophysical Journal International 202, 1 (2015), 119-141.

[21] Karve, P. M., Kallivokas, L. F., And Manuel, L. A framework for assessing the uncertainty in wave energy delivery to targeted subsurface formations. Journal of Applied Geophysics 125 (2016), 26-36.

[22] Karve, P. M., Kucukcoban, S., and Kallivokas, L. F. On an inverse source problem for enhanced oil recovery by wave motion maximization in reservoirs. Computational Geosciences 19 , 1 (2015), 233-256.

[23] Kucukcoban, S., Goh, H., And Kallivokas, L. F. On the full-waveform inversion of lame parameters in semi-infinite solids in plane strain. International Journal of Solids and Structures 164 (2019), $104-119$.

[24] Kucukcoban, S., And Kallivokas, L. F. Mixed perfectly-matched-layers for direct transient analysis in 2D elastic heterogeneous media. Computer Methods in Applied Mechanics and Engineering 200 (2011), 57-76. 
[25] Kucukcoban, S., And Kallivokas, L. F. A symmetric hybrid formulation for transient wave simulations in PML-truncated heterogeneous media. Wave Motion 50 (2013), 57 - 79.

[26] Lamert, A., Nguyen, L. T., Friederich, W., And Nestorovia, T. Imaging disturbance zones ahead of a tunnel by elastic full-waveform inversion: Adjoint gradient based inversion vs. parameter space reduction using a level-set method. Underground Space 3, 1 (2018), 21 - 33. Computational Methods in Mechanized Tunneling.

[27] LLOYD, F., AND JEONG, C. Adjoint equation-based inverse-source modeling to reconstruct moving acoustic sources in a 1D heterogeneous solid. Journal of Engineering Mechanics 144, 9 (2018), 04018089.

[28] Mashayekh, H., Kallivokas, L. F., And Tassoulas, J. L. Parameter estimation in layered media using dispersion-constrained inversion. Journal of Engineering Mechanics 144, 11 (2018), 04018099.

[29] Mejia, L., And Dawson, E. Earthquake deconvolution for FLAC. FLAC and Numerical (2006).

[30] Poul, M. K., AND ZERVA, A. Efficient time-domain deconvolution of seismic ground motions using the equivalent-linear method for soil-structure interaction analyses. Soil Dynamics and Earthquake Engineering 112 (2018), 138 - 151.

[31] Poul, M. K., AND ZERVA, A. Nonlinear dynamic response of concrete gravity dams considering the deconvolution process. Soil Dynamics and Earthquake Engineering 109 (2018), 324 - 338.

[32] Tadi, M., Rabitz, H., Sik, K. Y., Askar, A., Prevost, J. H., And McManus, J. B. Interior energy focusing within an elasto-plastic material. International Journal of Solids and Structures 33, 13 (1996), 1891-1901. 